J. Korean Math. Soc. 50 (2013), No. 4, pp. 695-713

http://dx.doi.org/10.4134/JKMS.2013.50.4.695

\title{
BIFURCATIONS OF A PREDATOR-PREY SYSTEM WITH WEAK ALLEE EFFECTS
}

\author{
Rongzhen Lin, Shengqiang Liu, and XiaOhong Lai
}

\begin{abstract}
We formulate and study a predator-prey model with nonmonotonic functional response type and weak Allee effects on the prey, which extends the system studied by Ruan and Xiao in [Global analysis in a predator-prey system with nonmonotonic functional response, SIAM J. Appl. Math. 61 (2001), no. 4, 1445-1472] but containing an extra term describing weak Allee effects on the prey. We obtain the global dynamics of the model by combining the global qualitative and bifurcation analysis. Our bifurcation analysis of the model indicates that it exhibits numerous kinds of bifurcation phenomena, including the saddle-node bifurcation, the supercritical and the subcritical Hopf bifurcations, and the homoclinic bifurcation, as the values of parameters vary. In the generic case, the model has the bifurcation of cusp type of codimension 2 (i.e., Bogdanov-Takens bifurcation).
\end{abstract}

\section{Introduction}

Since the pioneering work by Lotka and Volterra (see $[11,18]$ ), the predatorprey interaction models have been widely studied. We recall that in some cases the initial numbers of a species determine the long-term behavior of the system. Population models with incorporated Allee effects have such property. Allee effects, named after an ecologist, Warder C. Allee (see [1], [2], [3]), are defined as a positive relationship between any component of fitness of a species and either numbers or density of conspecifics (see [15]) meaning that an individual of a species that is subject to an Allee effects will suffer a decrease in some aspect of its fitness when conspecific density is low.

In some cases, this leads to a demographic Allee effects where the overall fitness has a positive relationship with density that results in per capita growth rate of the species being reduced at low density. A demographic Allee effects can be either weak or strong (see $[7,19])$. Populations subject to a strong Allee

Received December 17, 2009.

2010 Mathematics Subject Classification. 34C23, 92D25.

Key words and phrases. predator-prey, weak Allee effects, bifurcation, limit cycle.

The second author is supported by the National Natural Science Foundation of China (No. 10601042) and the Fundamental Research Funds the Central Universities (No. HIT.NSRIF.2010052) 
effects experience negative per capita growth rates when density falls below a critical threshold. Under deterministic dynamics, population that does not exceed this threshold will become extinct. Many studies consider only strong Allee effects but the examples in Allees original work show clearly that the definition should also include weak Allee effects (see [1], [2], [3], [8], [14], [15], [19], [20], [21], [26]). Populations with weak Allee dynamics experience lower per capita growth rates at low densities but never experience negative per capita growth rates and therefore have no critical threshold to exceed. We also refer to weak Allee effects as non-critical Allee effects and strong Allee effects as critical Allee effects. Strong Allee effects are termed with critical depensation and weak Allee effects with non-critical depensation (see [6]). Occasionally the term depensation is used to refer to population phenomena that do not arise from changes in individual fitness and therefore are not Allee effects (see [15]).

What is the influence of weak Allee effects in the interacting biological species? Only few papers have considered dynamical impacts of weak Allee effects on the predator-prey systems (see [9], [21], [27]). By modeling the case of resource subsidy and phase plane analysis, Kent et al. [9] concluded that the predator-prey systems stabilized by an influx of prey in the form of a rescue effect and destabilized by an outflux of a weak Allee effects; Wang and others $[21],[27]$ showed that weak Allee effects may be a destabilizing force in predatorprey systems.

In this paper, we introduced the weak Allee effects to the prey of a wellstudied predator-prey system with nonmonotonic functional responses to consider its dynamical behaviors. The mechanisms of nonmonotonic functional response is due to the group defense of prey against the predator, which is supported by many experiments (for details, we refer to [23], [24], [28]). Our work is motivated by that of Ruan and Xiao in [24], where the authors systematically considered the following predator-prey system with nonmonotonic functional response:

$$
\left\{\begin{array}{l}
\dot{x}(t)=r x\left(1-\frac{x}{K}\right)-\frac{m x y}{a+x^{2}}, \\
\dot{y}(t)=y\left(-d+\frac{u m x}{a+x^{2}}\right) .
\end{array}\right.
$$

In System (1), $x(t), y(t)$ are the prey and the predator density, respectively; $r$, $K, m, a, u>0 ; r$ denotes the intrinsic growth rate of the prey $x(t), K$ denotes the carrying capacity of the prey $x(t), d$ denotes the predator $y(t)$ 's natural death rate; the function $p(x)=\frac{m x}{a+x^{2}}$ is the nonmonotonic functional response. By choosing the carrying capacity of the prey and the death rate of the predator as bifurcation parameters, Ruan and Xiao showed in [24] that System (1) undergoes a series of bifurcations, including the saddle-node bifurcation, the 
supercritical and the subcritical Hopf bifurcations, and the homoclinic bifurcation. Further, by engaging the theories of Bogdanov [4], [5] and of Takens [17], the authors found that the system exhibits the Bogdanov-Takens bifurcation.

Based on System (1), by introducing the weak Allee effect to the prey $x$, we are able to construct our model as follows:

$$
\left\{\begin{array}{l}
\dot{x}(t)=r x\left(1-\frac{x}{K}\right) \frac{x}{x+A}-\frac{m x y}{a+x^{2}}, \\
\dot{y}(t)=y\left(-d+\frac{u m x}{a+x^{2}}\right) .
\end{array}\right.
$$

Here, $q(x)=\frac{x}{x+A}$ is the term for the weak Allee effect, where $A>0$ is defined as a "weak Allee effect constant" (see also [21]). It is easy to see that System (2) reduces to (1) as $A=0$ and that a larger $A$ means a stronger weak Allee effect.

The purpose of this paper is to study the global qualitative and bifurcation analysis and to obtain the influences of varying $A$ on the dynamics of (1). We refer the mathematical techniques to [22], [23], [24] and [28].

This paper is organized as follows. Section 2 contains the linear analysis of the equilibria and the general phase portraits analysis of equilibria. In Section 3 , we study the bifurcations. We determine that the parameter $r$ plays no role here.

\section{General phase portraits analysis of equilibria}

Model (2) involves seven parameters that all have biological interpretations. By rescaling the state variables and time, we can eliminate $a, m$, and $u$ by using

$$
(t, x, y / m) \rightarrow\left(\frac{\sqrt{a}}{m u} t, \sqrt{a} x, \sqrt{a} u y / m\right),
$$

and by resetting $r$ by $\frac{\sqrt{a} r}{m u}, K$ by $\frac{K}{\sqrt{a}}, A$ by $\frac{A}{\sqrt{a}}$, and $d$ by $\frac{\sqrt{a} d}{m u},(2)$ becomes

$$
\left\{\begin{array}{l}
\dot{x}(t)=r x\left(1-\frac{x}{K}\right) \frac{x}{x+A}-\frac{x y}{a+x^{2}}, \\
\dot{y}(t)=y\left(-d+\frac{u x}{a+x^{2}}\right) .
\end{array}\right.
$$

From the standpoint of biology, we are interested only in the dynamics of System (4) in the closed $\mathbb{R}_{+}^{2}$, thus we consider only the biologically meaningful initial conditions $x(0) \geq 0, y(0) \geq 0$.

First, we study the location and the number of the equilibrium solutions. There are two equilibria on the boundary of the nonnegative cone: $E_{0}=(0,0)$, representing extinctions of both species, and $E_{K}=(K, 0)$, representing extinction of the predator population and the density of the prey population equilibrating at the carrying capacity. 
System (4) has equilibria in the interior of $\mathbb{R}_{+}^{2}$ (coexistence equilibria) if and only if the equations

$$
\left\{\begin{array}{l}
r x\left(1-\frac{x}{K}\right) \frac{x}{x+A}-\frac{y}{a+x^{2}}=0, \\
-d+\frac{u x}{a+x^{2}}=0
\end{array}\right.
$$

have a pair of positive solutions $(x, y)$. It is clear that equation (5) have at most two pairs of solutions, $E_{1}=\left(x_{1}, y_{1}\right)$ and $E_{2}=\left(x_{2}, y_{2}\right)$, where

$$
\begin{aligned}
& x_{1}=\frac{u-\sqrt{u^{2}-4 a d^{2}}}{2 d}, y_{1}=\frac{r x_{1}\left(1-\frac{x_{1}}{K}\right)\left(a+x^{2}\right)}{x_{1}+A} ; \\
& x_{2}=\frac{u+\sqrt{u^{2}-4 a d^{2}}}{2 d}, y_{2}=\frac{r x_{2}\left(1-\frac{x_{2}}{K}\right)\left(a+x^{2}\right)}{x_{2}+A} .
\end{aligned}
$$

Therefore, we have the following simple lemma to describe the number and the location of equilibria of System (4). The proof is omitted.

Lemma 2.1. (1) When $\sqrt{u^{2}-4 a d^{2}}<0$, System (4) has two equilibria $E_{0}$, $E_{K}$ in $\mathbb{R}_{+}^{2}$.

(2) When $\sqrt{u^{2}-4 a d^{2}}=0$, System (4) has three equilibria in $\mathbb{R}_{+}^{2}, E_{0}, E_{K}$, $E_{12}=\left(x_{12}, y_{12}\right)$, where $x_{12}=\frac{u}{2 d}, \quad y_{12}=\frac{r x_{12}\left(1-\frac{x_{12}}{K}\right)\left(a+x^{2}\right)}{x_{12}+A}$.

(3) When $\sqrt{u^{2}-4 a d^{2}}>0$, then

System (4) has two equilibria in $\mathbb{R}_{+}^{2}, E_{0}, E_{K}$, if $K<x_{1}$;

System (4) has three equilibria in $\mathbb{R}_{+}^{2}, E_{0}, E_{K}, E_{1}$, if $x_{1}<K<x_{2}$;

System (4) has four equilibria in $\mathbb{R}_{+}^{2}, E_{0}, E_{K}, E_{1}, E_{2}$, if $x_{1}<x_{2}<K$.

When $\sqrt{u^{2}-4 a d^{2}}<0,-d+\frac{u x}{a+x^{2}}<0$ for all $x>0$, and $\dot{y}(t)<0$. Hence $\lim _{t \rightarrow \infty} y(t)\left(t, t_{0}, x_{0}, y_{0}\right)=0$ as $x_{0}>0, y_{0}>0$. This implies that the predator species goes extinct. Mathematically, $u^{2}-4 a d^{2}=0$ is a saddle-node bifurcation surface. When the parameters pass from one side of the surface to the other, the number of equilibria of the system changes as $u^{2}-4 a d^{2}>0$.

Now we consider the dynamics of System (4) in the neighborhood of each equilibrium. The linear part of System (4) at the equilibria $\left(x_{i}, y_{i}\right)$ is determined by

$$
V\left(x_{i}, y_{i}\right)=\left(\begin{array}{cc}
F\left(x_{i}, y_{i}\right) & -\frac{x_{i}}{a+x_{i}^{2}} \\
\frac{u y_{i}\left(a-x_{i}^{2}\right)}{\left(a+x_{i}^{2}\right)^{2}} & \frac{u x_{i}}{a+x_{i}^{2}}-d
\end{array}\right)_{\left(x_{i}, y_{i}\right)},
$$

where $F\left(x_{i}, y_{i}\right)=r\left[\left(1-\frac{x_{i}}{K}\right) \frac{x_{i}}{x_{i}+A}-\frac{x_{i}}{K} \frac{x_{i}}{x_{i}+A}+x_{i}\left(1-\frac{x_{i}}{K}\right) \frac{x_{i}}{\left(x_{i}+A\right)^{2}}\right]-\frac{a-x_{i}^{2}}{\left(a+x_{i}^{2}\right)^{2}} y_{i}$.

By studying the eigenvalues of the matrix $V(x, y)$ at the equilibrium, we can obtain the local dynamics of System (2) in the neighborhood of an equilibrium. We have:

$E_{0}$ is a saddle-node point where the parabolic sector is in $\mathbb{R}_{+}^{2}$.

$E_{K}$ is an attracting node if $-d+\frac{u K}{a+K^{2}}<0$ or a saddle point if $-d+\frac{u K}{a+K^{2}}>0$. If $-d+\frac{u K}{a+K^{2}}=0, E_{K}$ undergoes a transcritical bifurcation. 
For the interior equilibria $E_{1}, E_{2}$, since $\operatorname{det}\left(V\left(x_{2}, y_{2}\right)\right)=\frac{x_{2}}{a+x_{2}^{2}} \frac{u y_{2}\left(x_{2}^{2}\right)}{a+x_{2}^{2}}<0$, $E_{2}$ is a saddle point. Since $\operatorname{det}\left(V\left(x_{1}, y_{1}\right)\right)=\frac{x_{1}}{a+x_{1}^{2}} \frac{u y_{1}\left(x_{1}^{2}\right)}{a+x_{1}^{2}}>0$ and the trace of $V\left(x_{1}, y_{1}\right)$ is $\operatorname{tr}\left(V\left(x_{1}, y_{1}\right)\right)=F\left(x_{1}, y_{1}\right), E_{1}$ is an attractor if $F\left(x_{1}, y_{1}\right)<0$ and a repeller if $F\left(x_{1}, y_{1}\right)>0$.

As $d$ increases from the steady state, bifurcations outlined below occur.

1. Given $K<x_{12}$ and $-d+\frac{u K}{a+K^{2}}=0$, a transcritical bifurcation involving $E_{1}$ and $E_{K}$ occurs when $K=x_{1} . E_{K}$ changes its stability from an attracting node to a saddle point. When $u^{2}=4 a d^{2}$, a saddle-node bifurcation involving $E_{1}$ and $E_{2}$ occurs outside the nonnegative cone.

2. Given $K>x_{12}$ and $-d+\frac{u K}{a+K^{2}}=0$, a transcritical bifurcation involving $E_{2}$ and $E_{K}$ occurs when $K=x_{2} . E_{K}$ changes its stability from a saddle point to an attracting node. When $u^{2}=4 a d^{2}$, a saddle-node bifurcation involving $E_{1}$ and $E_{2}$ occurs outside the nonnegative cone.

3. Given $K=x_{12},-d+\frac{u K}{a+K^{2}}=0$ and $u^{2}=4 a d^{2}, E_{K}, E_{1}, E_{2}$ and $E_{12}$ coalesce at $E_{K}$. Phase portrait analysis shows that $E_{K}$ is an asymptotically stable degenerate node.

Remark 2.1. Comparing the above arguments to those on Model (1) by Ruan and Xiao [24], we see that the weak Allee effect constant $A$ does not affect the existence nor the number of the positive equilibria, but that an increase of $A$ will lower $y_{i}$ in the positive equilibria $E_{i}, i=1,2$ until they converge to the $y$-axis at the infinite value of $A$.

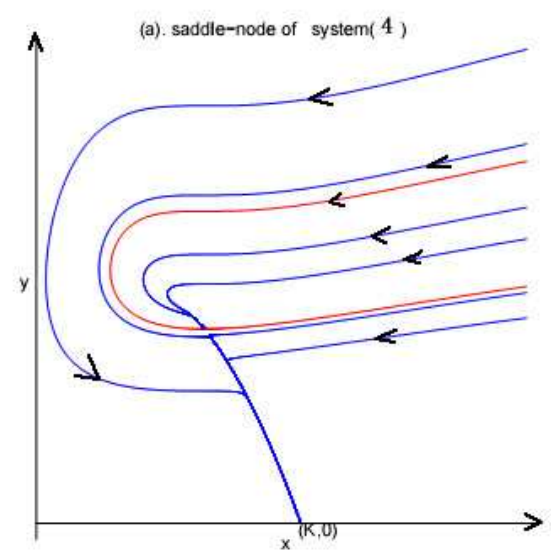

Figure 1. The phase portrait of System (4) when $u^{2}=4 a d^{2}$ and $\frac{u}{2 d}<K<\frac{u^{2}+3 u A d}{u d+4 A^{2} d}$. 


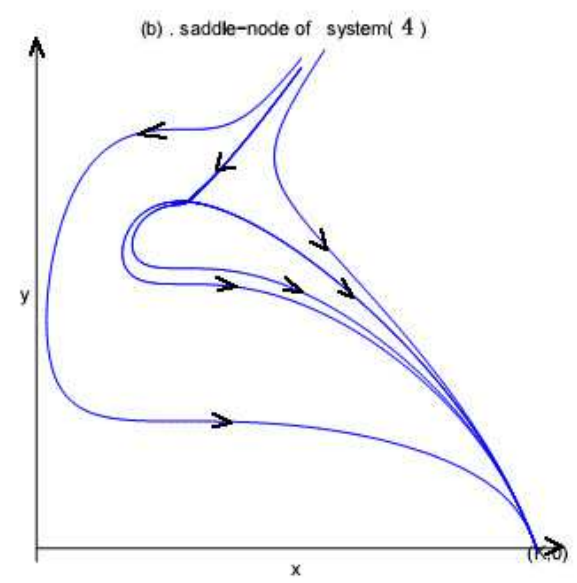

Figure 2. The phase portrait of System (4) when $u^{2}=4 a d^{2}$ and $K>\frac{u^{2}+3 u A d}{u d+4 A^{2} d}$.

Theorem 2.2. When $u^{2}=4 a d^{2}$ and $\frac{u}{2 d}<K$, System (4) has three equilibria, a saddle-node $E_{0}$, a stable node $E_{K}$, and an interior equilibrium $E_{12}$, where

(i) $E_{12}$ is a saddle-node consisting of two hyperbolic sectors and one parabolic sector; the parabolic sector is between the $y$-axis and the equilibrium if $K<$ $\frac{u^{2}+3 u A d}{u d+4 A^{2} d}$. The phase portrait is shown in Figure 1.

(ii) $E_{12}$ is a saddle-node consisting of two hyperbolic sectors and one parabolic sector; the hyperbolic sector is between the $y$-axis and the equilibrium if $K>\frac{u^{2}+3 u A d}{u d+4 A^{2} d}$. The phase portrait is shown in Figure 2.

(iii) $E_{12}$ is a cusp which consisting of two hyperbolic sectors and two seperatrices if $K=\frac{u^{2}+3 u A d}{u d+4 A^{2} d}$. One of the seperatrices converges to the interior equilibrium $E_{12}$ and all other solutions tend to the equilibrium $(K, 0)$. The phase portrait is shown in Figure 3.

Remark 2.2. In case (ii) of Theorem 2.2, we have similar results to those in 2.2 .2 [24]: the predator $y$ goes extinct in spite of the large carrying capacity $K$, i.e., the enrichment of the prey, which strongly supports the so-called "paradox of enrichment".

Proof of Theorem 2.2. We only prove case (iii). When $u^{2}=4 a d^{2}, K=\frac{u^{2}+3 u A d}{u d+4 A^{2} d}$, System (4) has a unique equilibrium $E_{12}$ located in the positive cone. Using a series of transformations, we reduce System (4) to the usual form.

The translation $X=x-x_{12}, Y=y-y_{12}$ brings $E_{12}$ to the origin $E_{0}$. Expanding the right-hand side of System (4) in a Taylor series about the origin, 


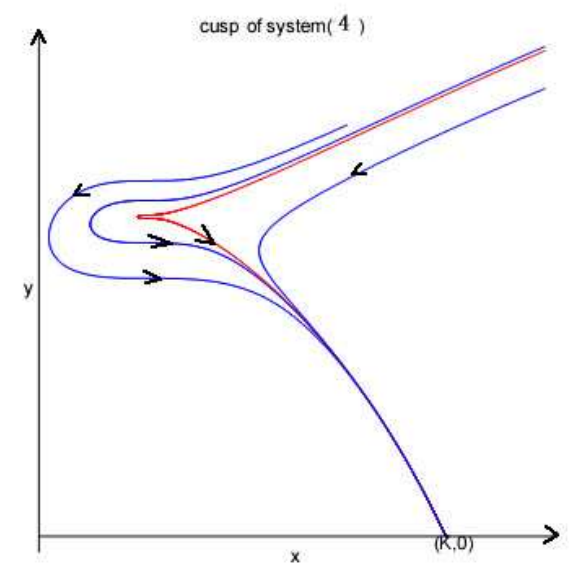

Figure 3. The phase portrait of System (4) when $u^{2}=4 a d^{2}$ and $K=\frac{u^{2}+3 u A d}{u d+4 A^{2} d}$.

we obtain

$(6)$

$$
\left\{\begin{aligned}
\dot{X}(t)= & -\frac{x_{12}}{a+x_{12}^{2}} Y+\left(-\frac{d r(u+6 A d)}{(u+2 A d)(u+3 A d)}+\frac{x_{12} y_{12}}{\left(a+x_{12}^{2}\right)^{2}}\right) X^{2} \\
& +P_{10}(X, Y) \\
\dot{Y}(t)= & \frac{u x_{12} y_{12}}{\left(a+x_{12}^{2}\right)^{2}} X^{2}+P_{20}(X, Y)
\end{aligned}\right.
$$

where $P_{i 0}(i=1,2)$ is $C^{\infty}$ in $(X, Y)$ and $P_{i 0}(X, Y)=O\left(|(X, Y)|^{3}\right)$.

Reversing time and making the transformation $X=X, Z=-\frac{x_{12}}{a+x_{12}^{2}} Y$, we have

$$
\left\{\begin{array}{l}
\dot{X}(t)=Z+\left(-\frac{d r(u+6 A d)}{(u+2 A d)(u+3 A d)}+\frac{x_{12} y_{12}}{\left(a+x_{12}^{2}\right)^{2}}\right) X^{2}+P_{11}(X, Z), \\
\dot{Z}(t)=\frac{u x_{12}^{2} y_{12}}{\left(a+x_{12}^{2}\right)^{3}} X^{2}+P_{21}(X, Z) .
\end{array}\right.
$$

Here, $P_{i 1}(i=1,2)$ is $C^{\infty}$ in $(X, Z)$ and $P_{i 1}(X, Z)=O\left(|(X, Z)|^{3}\right)$. Also, making the near-identity transformation

$$
\mu=X, \nu=Z+\left(-\frac{d r(u+6 A d)}{(u+2 A d)(u+3 A d)}+\frac{x_{12} y_{12}}{\left(a+x_{12}^{2}\right)^{2}}\right) X^{2}+P_{11}(X, Z),
$$

we obtain

$$
\left\{\begin{array}{l}
\dot{\mu}(t)=\nu \\
\dot{\nu}(t)=\delta_{1} \mu^{2}+\delta_{2} \mu \nu+P_{22}(\mu, \nu)
\end{array}\right.
$$


where $P_{22}$ is $C^{\infty}$ in $(\mu, \nu)$ and $P_{22}(\mu, \nu)=O\left(|(\mu, \nu)|^{3}\right)$,

$$
\begin{aligned}
\delta_{1} & =\frac{u x_{12}^{2} y_{12}}{\left(a+x_{12}^{2}\right)^{3}}=\frac{r d^{2}}{2(u+3 A d)}>0, \\
\delta_{2} & =2\left(-\frac{d r(u+6 A d)}{(u+2 A d)(u+3 A d)}+\frac{x_{12} y_{12}}{\left(a+x_{12}^{2}\right)^{2}}\right. \\
& =\frac{r d(-u-10 A d)}{2(u+2 A d)(u+3 A d)}<0 .
\end{aligned}
$$

Thus the interior equilibrium $E_{12}$ of System (4) is a cusp of codimension 2.

Next we discuss the dynamics of System (4) when $u^{2}>4 a d^{2}$ in detail. We know that $E_{1}$ is a stable focus (or node) when $F\left(x_{1}, y_{1}\right)<0$ and an unstable focus (or node) when $F\left(x_{1}, y_{1}\right)>0$. After an easy calculation, we obtain

$$
F\left(x_{1}, y_{1}\right)=\frac{r x_{1}}{K\left(x_{1}+A\right)}\left[K\left(2+\frac{A}{x_{1}+A}-\frac{2 a d}{u x_{1}}\right)-3 x_{1}-\frac{A x_{1}}{x_{1}+A}+\frac{2 a d}{u}\right] \text {. }
$$

Then the equation $F\left(x_{1}, y_{1}\right)=0$ holds true if $K=x_{1}+\frac{u x_{1}^{2}+A u x_{1}}{2 u x_{1}^{2}+3 A u x_{1}-2 a d x_{1}-2 a d A} x_{1}$.

For the sake of simplicity, we introduce $x_{3}$ :

$$
x_{3}=x_{1}+\frac{u x_{1}^{2}+A u x_{1}}{2 u x_{1}^{2}+3 A u x_{1}-2 a d x_{1}-2 a d A} x_{1} .
$$

It is easy to prove that $2 u x_{1}^{2}+3 A u x_{1}-2 a d x_{1}-2 a d A>0$ for all $x_{1}$ if $u^{2}>4 a d^{2}$. Then we have $x_{3}>x_{1}$. Therefore, we have:

Theorem 2.3. When $u^{2}>4 a d^{2}$,

(a) $E_{1}$ is a stable focus (or node) if $K<x_{3}$.

(b) $E_{1}$ is an unstable focus (or node) if $K>x_{3}$.

(c) $E_{1}$ is a weak focus or a center if $K=x_{3}$.

Considering the function $G(A)=x_{3}-x_{2}$, we have

$$
G(A)=\frac{u-\sqrt{u^{2}-4 a d^{2}}}{d}+\frac{\left(A u+u x_{1}\right) x_{1}^{2}}{2 u x_{1}^{2}+3 A u x_{1}-2 a d x_{1}-2 a d A}-\frac{u}{d} .
$$

Since $G(A)=0$ is equivalent to

$$
\begin{aligned}
& A\left(u d x_{1}^{2}-3 u x_{1} \sqrt{u^{2}-4 a d^{2}}+2 a d \sqrt{u^{2}-4 a d^{2}}\right) \\
= & \left(2 u x_{1} \sqrt{u^{2}-4 a d^{2}}-u d x_{1}^{2}-2 a d \sqrt{u^{2}-4 a d^{2}}\right) x_{1},
\end{aligned}
$$

we have:

when $u^{2}<\frac{13+5 \sqrt{17}}{8} a d^{2}$ (which is equivalent to $u d x_{1}^{2}-3 u x_{1} \sqrt{u^{2}-4 a d^{2}}+$ $\left.2 a d \sqrt{u^{2}-4 a d^{2}}>0\right), G(A)>0$, so $x_{3}>x_{2}$;

when $u^{2}>\frac{16}{3} a d^{2}$ (which is equivalent to $u d x_{1}^{2}-2 u x_{1} \sqrt{u^{2}-4 a d^{2}}+$ $\left.2 a d \sqrt{u^{2}-4 a d^{2}}<0\right), G(A)<0$, so $x_{3}<x_{2}$; 
when $\frac{13+5 \sqrt{17}}{8} a d^{2}<u^{2}<\frac{16}{3} a d^{2}$ (which is equivalent to $2 u x_{1} \sqrt{u^{2}-4 a d^{2}}<$ $\left.u d x_{1}^{2}+2 a d \sqrt{u^{2}-4 a d^{2}}<3 u x_{1} \sqrt{u^{2}-4 a d^{2}}\right)$, the sign of $G(A)$ is determined based on other conditions to be showed below.

For the sake of simplicity, we introduce $g, h$ :

$$
g=u d x_{1}^{2}+2 a d \sqrt{u^{2}-4 a d^{2}}, h=u x_{1} \sqrt{u^{2}-4 a d^{2}} .
$$

Then we have:

If $A>\frac{-g+2 h}{g-3 h}$, then $G(A)<0, x_{3}<x_{2}$;

If $A<\frac{-g+2 h}{g-3 h}$, then $G(A)>0, x_{3}>x_{2}$.

Now we summarize and classify the dynamics of System (4) in the interior of the first quadrant by dividing $u^{2}>4 a d^{2}$ into four cases: case (I) and case (II) are in Table 1, case (III) and case (IV) are in Table 2.

\begin{tabular}{|c|l|}
\hline \multicolumn{2}{|c|}{ Table 1 : (I) $u^{2}>\frac{16}{3} a d^{2}$ or } \\
$(\mathbf{I I}) \frac{13+5 \sqrt{17}}{8} a d^{2}<u^{2}<\frac{16}{3} a d^{2}, A>\frac{-g+2 h}{g-3 h}$ \\
\hline Range of parameter $K$ & Number of interior equilibria and stability of (4) \\
\hline$K<x_{1}$ & two equilibria: $E_{0}$ saddle-node, $E_{K}$ stable node \\
\hline$x_{1}<K<x_{3}$ & three equilibria: $E_{0}$ saddle-node, \\
& $E_{K}$ saddle, $E_{1}$ stable focus (or node) \\
\hline$x_{3}<K<x_{2}$ & three equilibria: $E_{0}$ saddle-node, $E_{K}$ saddle, \\
& $E_{1}$ unstable focus (or node) \\
\hline$K>x_{2}$ & four equilibria: $E_{0}$ saddle-node, $E_{K}$ stable node, \\
& $E_{1}$ unstable focus (or node), $E_{2}$ saddle \\
\hline \hline
\end{tabular}

\begin{tabular}{|c|l|}
\hline \multicolumn{2}{|c|}{ Table 2 : (III) $u^{2}<\frac{13+5 \sqrt{17}}{8} a d^{2}$ or } \\
$(\mathbf{I V}) \frac{13+5 \sqrt{17}}{8} a d^{2}<u^{2}<\frac{16}{3} a d^{2}, A<\frac{-g+2 h}{g-3 h}$ \\
\hline Range of parameter $K$ & Number of interior equilibria and stability of (4) \\
\hline$K<x_{1}$ & two equilibria: $E_{0}$ saddle-node, $E_{K}$ stable node \\
\hline$x_{1}<K<x_{2}$ & three equilibria: $E_{0}$ saddle-node, \\
& $E_{K}$ saddle, $E_{1}$ stable focus (or node) \\
\hline$x_{2}<K<x_{3}$ & four equilibria: $E_{0}$ saddle-node, $E_{K}$ saddle, \\
& $E_{1}$ stable focus (or node), $E_{2}$ saddle \\
\hline$K>x_{3}$ & four equilibria: $E_{0}$ saddle-node, $E_{K}$ stable node, \\
& $E_{1}$ unstable focus (or node), $E_{2}$ saddle \\
\hline \hline
\end{tabular}

Also, we have:

Theorem 2.4. If $u^{2}>\frac{16}{3} a d^{2}$ and $x_{3}<K<x_{2}$, then System (4) has at least one limit cycle in the interior of the first quadrant.

Proof. It is clear that the periodic orbit of System (4) must be in the domain $\Omega_{1}$ if it exists, where $\Omega_{1}=\{(x, y): 0<x<K, 0<y\}$. Taking a ray line $l_{1}$ 
beginning at the point $E_{K}, l_{1}=\{(x, y): x=K, y>0\}$ as we can see that the direction vector field of System (4) on $l_{1}$ is from right to left.

Now we consider the solution $\left(x^{*}(t), y^{*}(t)\right)$ of System (4) passing through the point $B\left(K, y_{b}\right)$, where $y_{b}>y_{1}$. It is easy to prove that the orbit $\left(x^{*}(t), y^{*}(t)\right)$ must cross the line $l_{2}$ at point $C\left(x_{1}, y_{c}\right)$ and $y_{c}>y_{b}$, where $l_{2}=\{(x, y): x=$ $\left.x_{1}, y>0\right\}$. Making a line $l_{3}=\left\{(x, y): y=y_{c}\right\}$, which begins at point $C\left(x_{1}, y_{c}\right)$ and ends at point $D\left(0, y_{c}\right)$, we can see that the direction vector field of System (4) on $l_{3}$ is from top to bottom (see Figure 4). Therefore, the orbits of System

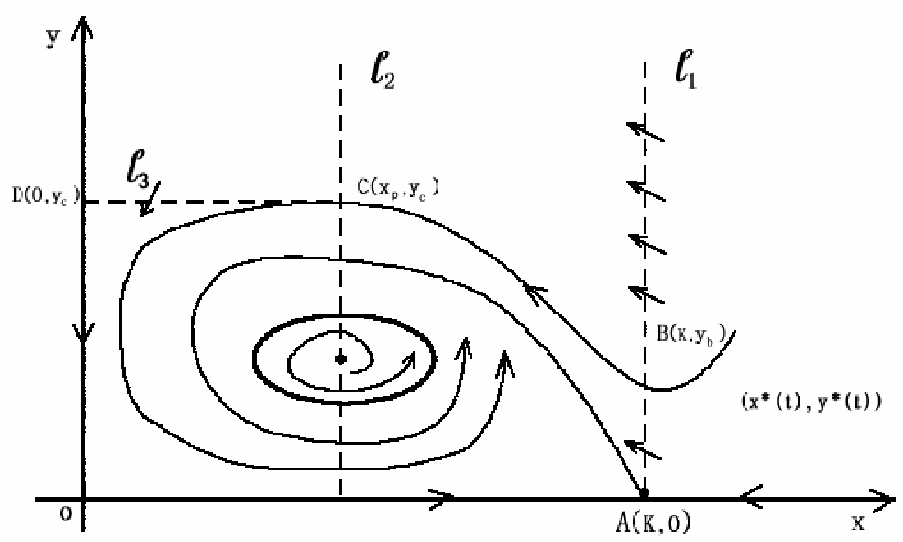

Figure 4. The phase portrait of System (4) when $u^{2}>\frac{16}{3} a d^{2}$ and $x_{3}<K<x_{2}$.

(4) in the interior of the region OABCDO cannot cross the boundary. On the other hand, we know that the equilibrium $\left(x_{1}, y_{1}\right)$ is an unstable focus (or node) since $K>x_{3}$. Therefore, the existence of a periodic orbit follows directly from the Poincare-Bendixson theorem. Moreover, the periodic orbit is stable in the interior.

Using the same method as in the proof of Theorem 2.4, we obtain the following theorems.

Theorem 2.5. If $\frac{13+5 \sqrt{17}}{8} a d^{2}<u^{2}<\frac{16}{3} a d^{2}, A>\frac{-g+2 h}{g-3 h} x_{1}$ and $x_{3}<K<x_{2}$, then System (4) has at least one limit cycle in the interior of the first quadrant.

Theorem 2.6. If $u^{2}<\frac{13+5 \sqrt{17}}{8} a d^{2}$ and $x_{1}<K<x_{2}$, then System (4) has no closed orbits. Thus the equilibrium $\left(x_{1}, y_{1}\right)$ is a globally stable focus (or node). 


\section{Bifurcations}

From Theorem 2.3, we have that in parameter space $(r, u, a, d, K, A)$, there exists a surface

$$
H=\left\{(r, u, a, d, K, A): u^{2}>4 a d^{2}, K=x_{3}\right\}
$$

such that System (4) has three equilibria $E_{0}, E_{K}$, and $E_{1}$ in $\mathbb{R}_{+}^{2}$ if $u^{2}<\frac{16}{3} a d^{2}$ or $\frac{13+5 \sqrt{17}}{8} a d^{2}<u^{2}<\frac{16}{3} a d^{2}, A>\frac{-g+2 h}{g-3 h} x_{1}$, where $E_{o}$ is a saddle-node, $E_{K}$ is a hyperbolic saddle and $E_{1}$ is a weak focus or a center. System (4) has four equilibria $E_{0}, E_{K}, E_{1}$, and $E_{2}$ in $\mathbb{R}_{+}^{2}$ if $u^{2}>\frac{13+5 \sqrt{17}}{8} a d^{2}$ or $\frac{13+5 \sqrt{17}}{8} a d^{2}<u^{2}<$ $\frac{16}{3} a d^{2}, A<\frac{-g+2 h}{g-3 h} x_{1}$, where $E_{o}$ is a saddle-node, $E_{K}$ is a stable node, $E_{1}$ is a weak focus or a center, and $E_{2}$ is a hyperbolic saddle. Hence System (4) may undergo Hopf bifurcation.

In this section, we discuss the conditions under which the stability of $\left(x_{1}, y_{1}\right)$ changes so that System (4) exhibits Hopf bifurcation. We first determine the stability of the equilibrium $\left(x_{1}, y_{1}\right)$ when parameter $(r, u, a, d, K, A)$ belongs to $H$. In order to observe the stability, we need to compute the Lyapunov coefficients of $\left(x_{1}, y_{1}\right)$. Making the transformation $X=x-x_{1}, Y=y-y_{1}$ to translate $\left(x_{1}, y_{1}\right)$ to the origin and rewriting $X$ and $Y$ as $x$ and $y$, respectively, we have

$$
\left\{\begin{aligned}
\dot{x}= & a_{10} x+a_{01} y+a_{20} x^{2}+a_{11} x y+a_{02} y^{2}+a_{30} x^{3} \\
& +a_{21} x^{2} y+a_{12} x y^{2}+a_{03} y^{3}+O_{1}\left(|(x, y)|^{4}\right), \\
\dot{y}= & b_{10} x+b_{01} y+b_{20} x^{2}+b_{11} x y+b_{02} y^{2}+b_{30} x^{3} \\
& +b_{21} x^{2} y+b_{12} x y^{2}+b_{03} y^{3}+O_{2}\left(|(x, y)|^{4}\right),
\end{aligned}\right.
$$

where $a_{i j}$ and $b_{i j}$ are the coefficients of the power series expansions of $r x(1-$ $\left.\frac{x}{K}\right) \frac{x}{x+A}-\frac{x y}{a+x^{2}}$ and $-d+\frac{u m x}{a+x^{2}}$ at $\left(x_{1}, y_{1}\right)$, respectively, $(i, j=0,1,2,3, \ldots)$, and $O_{k}\left(|(x, y)|^{4}\right)$ is the smooth function with at least the forth order with respect to $(x, y)(k=1,2)$. Then when parameter $(r, u, a, d, K, A)$ belongs to $H$, we have:

$$
\begin{aligned}
a_{10}= & -\frac{\left(a-x_{1}^{2}\right) y_{1}}{\left(a+x_{1}^{2}\right)^{2}}-\frac{r x_{1}}{K} \frac{x_{1}}{x_{1}+A}+2 r\left(1-\frac{x_{1}}{K}\right) \frac{x_{1}}{x_{1}+A} \\
& -r x_{1}\left(1-\frac{x_{1}}{K}\right) \frac{x_{1}}{x_{1}+A}=0 ; \\
a_{20}= & \frac{x_{1} y_{1}\left(3 a-x_{1}^{2}\right)}{\left(a+x_{1}^{2}\right)^{3}}-2 \frac{r x_{1}}{K} \frac{1}{x_{1}+A}+r\left(1-\frac{x_{1}}{K}\right) \frac{x_{1}}{x_{1}+A} \\
& -\frac{r x_{1}\left(-2 x_{1}^{2}-3 A x_{1}^{2}+K x_{1}+2 K A\right)}{K\left(x_{1}+A\right)^{3}} ; \\
a_{30}= & \frac{\left(x_{1}^{4}-6 a x_{1}^{2}+a^{2}\right) y_{1}}{\left(a+x_{1}^{2}\right)^{4}}-\frac{x_{1}}{K\left(x_{1}+A\right)}-\frac{r\left(-x_{1}^{3}-3 A x_{1}^{2}+3 A^{2} x_{1}+K A^{2}\right)}{K\left(x_{1}+A\right)^{4}} ;
\end{aligned}
$$




$$
\begin{aligned}
& a_{01}=-\frac{x_{1}}{a+x_{1}^{2}}, \quad a_{02}=0, \quad a_{03}=0, a_{11}=-\frac{a-x_{1}^{2}}{\left(a+x_{1}^{2}\right)^{2}}, \\
& a_{21}=-\frac{x_{1}\left(3 a-x_{1}^{2}\right)}{\left(a+x_{1}^{2}\right)^{3}}, \quad a_{12}=0, \\
& b_{10}=\frac{u\left(a-x_{1}^{2}\right) y_{1}}{\left(a+x_{1}^{2}\right)^{2}}, b_{20}=-\frac{u x_{1} y_{1}\left(3 a-x_{1}^{2}\right)}{\left(a+x_{1}^{2}\right)^{3}}, b_{11}=\frac{u\left(a-x_{1}^{2}\right)}{\left(a+x_{1}^{2}\right)^{2}} ; \\
& b_{30}=-\frac{u y_{1}\left(x_{1}^{4}-6 a x_{1}^{2}+a^{2}\right)}{\left(a+x_{1}^{2}\right)^{4}}, b_{21}=-\frac{u x_{1}\left(3 a-x_{1}^{2}\right)}{\left(a+x_{1}^{2}\right)^{3}}, b_{01}=0, b_{02}=0 .
\end{aligned}
$$

Hence using the formula of the first Lyapunov number $\sigma$ at the origin of (10) in [12], we have

Here,

$$
\sigma=-\frac{3 \pi}{2 \Delta^{\frac{2}{3}}} Q
$$

$$
\begin{aligned}
\Delta= & \frac{u x_{1} y_{1}\left(a-x_{1}^{2}\right)}{\left(a+x_{1}^{2}\right)^{3}} \\
Q= & -\frac{x_{1}}{a+x_{1}^{2}} \frac{u x_{1} y_{1}\left(3 a-x_{1}^{2}\right)}{\left(a+x_{1}^{2}\right)^{3}}\left(\frac{2 x_{1} y_{1}\left(3 a-x_{1}^{2}\right)}{\left(a+x_{1}^{2}\right)^{3}}\right. \\
& \left.+\frac{2 r\left(-x_{1}^{3}-3 A x_{1}^{2}-3 A^{2} x_{1}+K A\right)}{K\left(x_{1}+A\right)^{3}}+\frac{u\left(a-x_{1}^{2}\right)}{\left(a+x_{1}^{2}\right)^{2}}\right) \\
& +\frac{u\left(a-x_{1}^{2}\right) y_{1}}{\left(a+x_{1}^{2}\right)^{2}}\left[-\frac{3 x_{1}}{a+x_{1}^{2}}\left(\frac{\left(x_{1}^{4}-6 a x_{1}^{2}+a^{2}\right) y_{1}}{\left(a+x_{1}^{2}\right)^{4}}-\frac{r A^{2}(K+A)}{K\left(x_{1}+A\right)^{4}}\right)\right. \\
& +\frac{x_{1}}{a+x_{1}^{2}} \frac{u x_{1}\left(3 a-x_{1}^{2}\right)}{\left(a+x_{1}^{2}\right)^{3}} \\
& \left.+\frac{a-x_{1}^{2}}{\left(a+x_{1}^{2}\right)^{2}}\left(\frac{x_{1} y_{1}\left(3 a-x_{1}^{2}\right)}{\left(a+x_{1}^{2}\right)^{3}}+\frac{r\left(-x_{1}^{3}-3 A x_{1}^{2}-3 A^{2} x_{1}+K A\right)}{K\left(x_{1}+A\right)^{3}}\right)\right] .
\end{aligned}
$$

Therefore, the sign of $\sigma$ is determined by $Q$. If $Q \neq 0$, then the origin of (10) is a weak focus of multiplicity one that is stable if $Q<0$ and unstable if $Q>0$.

Using the fact that $x_{1}=\frac{u-\sqrt{u^{2}-4 a d^{2}}}{2 d}, y_{1}=\frac{r x_{1}\left(1-\frac{x_{1}}{x_{1}}\right)\left(a+x^{2}\right)}{x_{1}+A}$ with the aid of numerical calculations, we can see that the sign of $\sigma$ is not determined. For example, when the parameter $(r, u, a, d, K, A)=(1,2,0.3,1.3,0.6099181820,0.1)$ is on the surface $H$, then $Q=-1.036150079$. On the other hand, when the parameter $(r, u, a, d, K, A)=(1,5,0.5,1.2,0.2695986091,0.46)$ is also on the surface $H$, then $Q=0.3913608342$. Therefore, on the surface $H$, there exists a curve

$$
\ell=\left\{(r, u, a, d, K, A): Q=0, u^{2}>4 a d^{2}, K=x_{3}\right\}
$$

such that $\sigma=0$ since $\sigma$ is a continuous function of $(r, u, a, d, K, A)$. When parameter $(r, u, a, d, K, A)$ is on the curve l, the origin of (10) is a weak focus with multiplicity of at least two or a center. Hence the surface $H$ is divided 
into two parts, $H_{b}$ and $H_{p}$, by the curve $\ell, \sigma>0$ if $(r, u, a, d, K, A)$ is in $H_{b}$ and $\sigma<0$ if $(r, u, a, d, K, A)$ is in $H_{p}$. That is,

$$
\begin{aligned}
& H_{b}=\left\{(r, u, a, d, K, A): u^{2}>4 a d^{2}, K=x_{3}, Q<0\right\}, \\
& H_{p}=\left\{(r, u, a, d, K, A): u^{2}>4 a d^{2}, K=x_{3}, Q>0\right\} .
\end{aligned}
$$

Summarizing the above discussions, we obtain:

Theorem 3.1. (a) If the parameter $(r, u, a, d, K, A)$ is in $H_{b}$, then the equilibrium $\left(x_{1}, y_{1}\right)$ of System (4) is a weak focus of multiplicity one and is unstable.

(b) If the parameter $(r, u, a, d, K, A)$ is on the curve $\ell$, then the equilibrium $\left(x_{1}, y_{1}\right)$ of System (4) is a weak focus with multiplicity of at least two or a center.

(c) If the parameter $(r, u, a, d, K, A)$ is in $H_{p}$, then the equilibrium $\left(x_{1}, y_{1}\right)$ of System (4) is a weak focus of multiplicity one and is stable.

From Theorem 2.3 and the third case (c) in Theorem 3.1, we know that the weak focus $\left(x_{1}, y_{1}\right)$ generates a stable limit cycle as $K$ passes through the bifurcation value $K=x_{3}$. From one side of the surface $H_{p}$ to the other, System (10) may undergo a supercritical Hopf bifurcation (see [12]). A stable limit cycle appears in the small neighborhood of $\left(x_{1}, y_{1}\right)$ when $(r, u, a, d, K, A) \in H_{p}$, $K>x_{3}$ and $\left|K-x_{3}\right| \ll 1$. The surface $H_{p}$ is called a supercritical Hopf bifurcation.

On the other hand, from Theorem 2.3 and the first case (a) in Theorem 3.4, we know that the weak focus $\left(x_{1}, y_{1}\right)$ generates an unstable limit cycle as $K$ passes through the bifurcation value $K=x_{3}$. From one side of the surface $H_{b}$ to the other, System (10) may undergo a subcritical Hopf bifurcation (see [12]). A stable limit cycle appears in the small neighborhood of $\left(x_{1}, y_{1}\right)$ when $(r, u, a, d, K, A) \in H_{b}, K<x_{3}$ and $\left|K-x_{3}\right| \ll 1$. The surface $H_{b}$ is called a subcritical Hopf bifurcation.

By the above arguments, we have:

Theorem 3.2. (i) System (4) has at least one unstable limit cycle if $(r, u, a, d$, $K, A) \in H_{b}, 0<K<x_{3}$ and $\left|K-x_{3}\right| \ll 1$;

(ii) System (4) has at least one stable limit cycle if $(r, u, a, d, K, A) \in H_{p}$, $K>x_{3}$ and $\left|K-x_{3}\right| \ll 1$.

Remark 3.1. Since there exist some parameter values such that $\sigma=0$, System (4) may undergo a degenerate Hopf bifurcation for some parameter values. It is possible that there exist two limit cycles of System (4) in $\mathbb{R}_{+}^{2}$.

By Theorem 2.2, if $K=\frac{u^{2}+3 u A d}{u d+4 A^{2} d}$ and $u^{2}=4 a d^{2}$, then the unique equilibrium $E_{12}$ in the positive cone is a cusp point of codimension 2 . In this section, we are interested in the bifurcation of the cusp as the parameters vary in a small neighborhood of $\left(u_{0}, K_{0}, a_{0}, d_{0}, A_{0}\right)$, where $u_{0}, K_{0}, a_{0}, d_{0}$, and $A_{0}$ satisfy the equations

$$
u_{0}^{2}=4 a_{0} d_{0}^{2}, \quad K_{0}=\frac{u_{0}^{2}+3 u_{0} A_{0} d_{0}}{u_{0} d_{0}+4 A_{0}^{2} d_{0}}
$$


In the following arguments, we will find the universal unfolding of $E_{12}$ depending on the original parameters in System (4). We take $(K, d)$ as bifurcation parameters and develop a universal unfolding for the codimension 2 cusp singularity when these two parameters are perturbed near the point $\left(K_{0}, d_{0}\right)$, and System (4) can exhibit Bogdanov-Takens bifurcation. Let $(K, d)$ in a neighborhood of $\left(K_{0}, d_{0}\right)$ and

$$
\left\{\begin{array}{l}
K=K_{0}+\varepsilon_{1}, \\
d=d_{0}+\varepsilon_{2}
\end{array}\right.
$$

in (4). We study the bifurcations of the resulting system

$$
\left\{\begin{array}{l}
\dot{x}(t)=r x\left(1-\frac{x}{K_{0}+\varepsilon_{1}}\right) \frac{x}{x+A_{0}}-\frac{x y}{a_{0}+x^{2}}, \\
\dot{y}(t)=y\left(-\left(d_{0}+\varepsilon_{2}\right)+\frac{u_{0} x}{a_{0}+x^{2}}\right) .
\end{array}\right.
$$

For $\varepsilon=\left(\varepsilon_{1}, \varepsilon_{2}\right)$ sufficiently small, we have:

Theorem 3.3. For parameters $\varepsilon=\left(\varepsilon_{1}, \varepsilon_{2}\right)$ sufficiently small, System (4) with the conditions in (11) is a generic unfolding of the cusp singularity of codimension 2.

Proof. It is shown in [12] that a generic unfolding with the parameters $\left(\nu_{1}, \nu_{2}\right)$ of the codimension 2 cusp singularity is $C^{\infty}$ equivalent to

$$
\left\{\begin{array}{l}
\dot{x}(t)=y \\
\dot{y}(t)=\nu_{1}+\lambda \nu_{2} y+x^{2}+\lambda x y .
\end{array}\right.
$$

Using the method and results of [12], [24], [4] and [5], we will show that System (13) with parameters $\left(\varepsilon_{1}, \varepsilon_{2}\right)$ is also a generic unfolding of the codimension 2 singularity by showing that there exist smooth coordinate changes that take (13) into (14).

System (13) has a cusp point at $\left(x_{12}, y_{12}\right)=\left(\frac{u_{0}}{2 d_{0}}, \frac{r x_{12}\left(1-\frac{x_{12}}{K_{0}}\right)\left(a+x^{2}\right)}{x_{12}+A_{0}}\right)$ if $\varepsilon=$ $(0,0)$. Applying the translation

$$
X=x-x_{12}, \quad Y=y-y_{12}
$$

and expanding System (13) in the power series about the origin, we have (15)

$$
\left\{\begin{array}{l}
\dot{X}(t)=L_{10}\left(\varepsilon_{1}\right)+X L_{11}\left(\varepsilon_{1}\right)+Y L_{12}\left(\varepsilon_{1}\right)+X^{2} L_{13}\left(\varepsilon_{1}\right)+R_{10}\left(X, Y, \varepsilon_{1}\right), \\
\dot{Y}(t)=L_{20}\left(\varepsilon_{2}\right)+X L_{21}\left(\varepsilon_{1}\right)+Y L_{22}\left(\varepsilon_{2}\right)+X^{2} L_{23}\left(\varepsilon_{2}\right)+R_{20}\left(X, Y, \varepsilon_{2}\right),
\end{array}\right.
$$

and

$$
\begin{aligned}
& L_{10}\left(\varepsilon_{1}\right)=\frac{r x_{12}^{2}}{K_{0}^{2}} \frac{x_{12}}{A+x_{12}} \varepsilon_{1}+b_{1}\left(\varepsilon_{1}\right) \\
& L_{11}\left(\varepsilon_{1}\right)=\frac{r x_{12}^{2}}{K_{0}^{2}}\left(\frac{3}{A+x_{12}}-\frac{x_{12}}{\left(A+x_{12}\right)^{2}}\right) \varepsilon_{1}+b_{2}\left(\varepsilon_{1}\right),
\end{aligned}
$$




$$
\begin{aligned}
L_{12}\left(\varepsilon_{1}\right)= & \frac{x_{12}}{2 a_{0}}, \\
L_{13}\left(\varepsilon_{1}\right)= & \frac{x_{12} y_{12}}{4 a_{0}^{2}}-\frac{d_{0} r_{0}\left(u_{0}+6 A_{0} d_{0}\right)}{\left(u_{0}+2 A_{0} d_{0}\right)\left(u_{0}+3 A_{0} d_{0}\right)} \\
& +\frac{r x_{12}}{K_{0}^{2}\left(A_{0}+x_{12}\right)}\left(\frac{x_{12}^{2}}{\left(A_{0}+x_{12}\right)^{2}}+\frac{x_{12}}{\left(A_{0}+x_{12}\right)}-1\right) \varepsilon_{1}+b_{3}\left(\varepsilon_{1}\right), \\
L_{20}\left(\varepsilon_{2}\right)= & -y_{12} \varepsilon_{2}, L_{21}\left(\varepsilon_{2}\right)=0, L_{22}\left(\varepsilon_{2}\right)=-\varepsilon_{2}, L_{23}\left(\varepsilon_{2}\right)=-\frac{u_{0} x_{12} y_{12}}{\left(a_{0}+x_{12}^{2}\right)^{2}},
\end{aligned}
$$

where $b_{i}\left(\varepsilon_{i}\right)(i=1,2,3)$ are smooth functions of at least the second order, $R_{10}$ and $R_{20}$ are $C^{\infty}$ functions of at least the third order with respect to $(X, Y)$, and the coefficients depend smoothly on $\varepsilon_{1}$ and $\varepsilon_{2}$, respectively.

By the transformation

$$
X=X, Z=\left(\frac{r x_{12}^{2}}{K_{0}^{2}}\left(\frac{3}{A+x_{12}}-\frac{x_{12}}{\left(A+x_{12}\right)^{2}}\right) \varepsilon_{1}+b_{2}\left(\varepsilon_{1}\right)\right) X-\frac{x_{12}}{2 a_{0}} Y,
$$

System (15) is $C^{\infty}$ equivalent to

$$
\left\{\begin{aligned}
\dot{X}(t)= & \bar{L}_{10}\left(\varepsilon_{1}\right)+X \bar{L}_{11}\left(\varepsilon_{1}\right)+Z \bar{L}_{12}\left(\varepsilon_{1}\right)+X^{2} \bar{L}_{13}\left(\varepsilon_{1}\right) \\
& +\bar{R}_{10}\left(X, Z, \varepsilon_{1}\right) \\
\dot{Z}(t)= & \bar{L}_{20}\left(\varepsilon_{1}, \varepsilon_{2}\right)+X \bar{L}_{21}\left(\varepsilon_{1}, \varepsilon_{1}\right)+Z \bar{L}_{22}\left(\varepsilon_{1}, \varepsilon_{2}\right) \\
& +X^{2} \bar{L}_{23}\left(\varepsilon_{1}, \varepsilon_{2}\right)+\bar{R}_{20}\left(X, Z, \varepsilon_{2}\right)
\end{aligned}\right.
$$

and

$$
\begin{aligned}
\bar{L}_{10}\left(\varepsilon_{1}\right)= & \frac{r x_{12}^{2}}{K_{0}^{2}} \frac{x_{12}}{A+x_{12}} \varepsilon_{1}+b_{1}\left(\varepsilon_{1}\right), \bar{L}_{11}\left(\varepsilon_{1}\right)=0, \bar{L}_{12}\left(\varepsilon_{1}\right)=1, \\
\bar{L}_{13}\left(\varepsilon_{1}\right)= & \frac{x_{12} y_{12}}{4 a_{0}^{2}}-\frac{d_{0} r_{0}\left(u_{0}+6 A_{0} d_{0}\right)}{\left(u_{0}+2 A_{0} d_{0}\right)\left(u_{0}+3 A_{0} d_{0}\right)} \\
& +\frac{r x_{12}}{K_{0}^{2}\left(A_{0}+x_{12}\right)}\left(\frac{x_{12}^{2}}{\left(A_{0}+x_{12}\right)^{2}}+\frac{x_{12}}{\left(A_{0}+x_{12}\right)}-1\right) \varepsilon_{1}+b_{3}\left(\varepsilon_{1}\right), \\
\bar{L}_{20}\left(\varepsilon_{1}, \varepsilon_{2}\right)= & \frac{x_{12} y_{12}}{2 a_{0}} \varepsilon_{2}+c_{1}\left(\varepsilon_{1}\right), \bar{L}_{21}\left(\varepsilon_{1}, \varepsilon_{2}\right)=c_{2}\left(\varepsilon_{1}\right), \\
\bar{L}_{22}\left(\varepsilon_{1}, \varepsilon_{2}\right)= & \frac{r x_{12}^{2}}{K_{0}^{2}} \frac{3 A+2 x_{12}}{\left(A+x_{12}\right)^{2}} \varepsilon_{1}-\varepsilon_{2}+b_{2}\left(\varepsilon_{1}\right), \\
\bar{L}_{23}\left(\varepsilon_{1}, \varepsilon_{2}\right)= & -\frac{u_{0} x_{12}^{2} y_{12}}{2 a_{0}\left(a_{0}+x_{12}^{2}\right)^{2}}+c_{3}\left(\varepsilon_{1}\right),
\end{aligned}
$$

where $\bar{R}_{10}$ and $\bar{R}_{20}$ are $C^{\infty}$ functions in variables $(X, Z)$ of at least the third order, the coefficients depend smoothly on $\varepsilon_{1}$ and $\varepsilon_{2}$, and $c_{1}, c_{2}$, and $c_{3}$ are smooth functions of their variables. Let

$X=X$,

$\nu=\bar{L}_{20}\left(\varepsilon_{1}, \varepsilon_{2}\right)+X \bar{L}_{21}\left(\varepsilon_{1}, \varepsilon_{1}\right)+Z \bar{L}_{22}\left(\varepsilon_{1}, \varepsilon_{2}\right)+X^{2} \bar{L}_{23}\left(\varepsilon_{1}, \varepsilon_{2}\right)+\bar{R}_{20}\left(X, Z, \varepsilon_{2}\right)$. 
Thus System (16) becomes

$$
\left\{\begin{aligned}
\dot{X}(t)= & \nu \\
\dot{\nu}(t)= & \widetilde{L}_{20}\left(\varepsilon_{1}, \varepsilon_{2}\right)+X \widetilde{L}_{21}\left(\varepsilon_{1}, \varepsilon_{2}\right)+\nu \widetilde{L}_{22}\left(\varepsilon_{1}, \varepsilon_{2}\right) \\
& +X^{2} \widetilde{L}_{23}\left(\varepsilon_{1}, \varepsilon_{2}\right)+X \nu \widetilde{L}_{24}\left(\varepsilon_{1}, \varepsilon_{2}\right)+\widetilde{R}_{20}\left(X, Y, \varepsilon_{1}, \varepsilon_{2}\right)
\end{aligned}\right.
$$

where

$$
\begin{aligned}
\widetilde{L}_{20}\left(\varepsilon_{1}, \varepsilon_{1}\right)= & \frac{x_{12} y_{12}}{2 a_{0}} \varepsilon_{2}+\widetilde{c}_{1}\left(\varepsilon_{1}, \varepsilon_{2}\right), \widetilde{L}_{21}\left(\varepsilon_{1}, \varepsilon_{2}\right)=\widetilde{c}_{2}\left(\varepsilon_{1}, \varepsilon_{2}\right), \\
\widetilde{L}_{22}\left(\varepsilon_{1}, \varepsilon_{2}\right)= & \frac{r x_{12}^{2}}{K_{0}^{2}} \frac{3 A+2 x_{12}}{\left(A+x_{12}\right)^{2}} \varepsilon_{1}-\varepsilon_{2}+b_{2}\left(\varepsilon_{1}\right), \\
\widetilde{L}_{23}\left(\varepsilon_{1}, \varepsilon_{2}\right)= & \frac{r u_{0}}{8 a_{0}} \frac{u_{0}}{u_{0}+3 A_{0} d_{0}}+\widetilde{c}_{3}\left(\varepsilon_{1}, \varepsilon_{2}\right), \\
\widetilde{L}_{24}\left(\varepsilon_{1}, \varepsilon_{2}\right)= & \frac{d_{0} r_{0}\left(u_{0}+10 A_{0} d_{0}\right)}{2\left(u_{0}+2 A_{0} d_{0}\right)\left(u_{0}+3 A_{0} d_{0}\right)} \\
& -\frac{r x_{12}}{K_{0}^{2}\left(A_{0}+x_{12}\right)}\left(\frac{x_{12}^{2}}{\left(A_{0}+x_{12}\right)^{2}}+\frac{x_{12}}{\left(A_{0}+x_{12}\right)}-1\right) \varepsilon_{1}+b_{3}\left(\varepsilon_{1}\right),
\end{aligned}
$$

and $\widetilde{c}_{i}(\mathrm{i}=1,2,3)$ are smooth functions of $\left(\varepsilon_{1}, \varepsilon_{2}\right) ; \widetilde{R}_{20}$ is a $C^{\infty}$ function in variables $\left(\varepsilon_{1}, \varepsilon_{2}\right)$ of at least the third order with respect to $(X, \nu)$, and the coefficients depend smoothly on $\varepsilon_{1}$ and $\varepsilon_{2}$. Then by the transform

$$
\mu=X, v=\frac{\nu}{\sqrt{\widetilde{L}_{23}\left(\varepsilon_{1}, \varepsilon_{2}\right)}}, \tau=\int_{0}^{t} \sqrt{\widetilde{L}_{23}\left(\varepsilon_{1}, \varepsilon_{2}\right)} \mathrm{d} s
$$

we have

$$
\left\{\begin{aligned}
\dot{\mu}(t)= & v \\
\dot{v}(t)= & \widetilde{\widetilde{L}}_{20}\left(\varepsilon_{1}, \varepsilon_{2}\right)+\mu \widetilde{\widetilde{L}}_{21}\left(\varepsilon_{1}, \varepsilon_{2}\right)+v \widetilde{\widetilde{L}}_{22}\left(\varepsilon_{1}, \varepsilon_{2}\right) \\
& +\mu^{2} \widetilde{\widetilde{L}}_{23}\left(\varepsilon_{1}, \varepsilon_{2}\right)+\mu v \widetilde{\widetilde{L}}_{24}\left(\varepsilon_{1}, \varepsilon_{2}\right)+\widetilde{\widetilde{R}}_{20}\left(\mu, v, \varepsilon_{1}, \varepsilon_{2}\right)
\end{aligned}\right.
$$

where

$$
\begin{aligned}
\widetilde{\widetilde{L}}_{20}\left(\varepsilon_{1}, \varepsilon_{2}\right) & =\frac{x_{12} y_{12}}{2 a_{0}\left(\frac{r u_{0}}{8 a_{0}} \frac{u_{0}}{u_{0}+3 A_{0} d_{0}}+\widetilde{c}_{3}\left(\varepsilon_{1}, \varepsilon_{2}\right)\right.} \varepsilon_{2}+\frac{\widetilde{c}_{1}\left(\varepsilon_{1}, \varepsilon_{2}\right)}{\frac{r u_{0}}{8 a_{0}} \frac{u_{0}}{u_{0}+3 A_{0} d_{0}}+\widetilde{c}_{3}\left(\varepsilon_{1}, \varepsilon_{2}\right)} \\
& =\frac{4 a_{0} x_{12}}{u_{0}} \varepsilon_{2}+\phi_{1}\left(\varepsilon_{1}, \varepsilon_{2}\right), \\
\widetilde{L}_{21}\left(\varepsilon_{1}, \varepsilon_{2}\right) & =\frac{\widetilde{c}_{2}\left(\varepsilon_{1}, \varepsilon_{2}\right)}{\frac{r u_{0}}{8 a_{0}} \frac{u_{0}}{u_{0}+3 A_{0} d_{0}}+\widetilde{c}_{3}\left(\varepsilon_{1}, \varepsilon_{2}\right)}=\phi_{2}\left(\varepsilon_{1}, \varepsilon_{2}\right), \\
\widetilde{\widetilde{L}}_{22}\left(\varepsilon_{1}, \varepsilon_{2}\right) & =\frac{\frac{r x_{12}^{2}}{K_{0}^{2}} \frac{3 A+2 x_{12}}{\left(A+x_{12}\right)^{2}} \varepsilon_{1}-\varepsilon_{2}+b_{2}\left(\varepsilon_{1}\right)}{\sqrt{\frac{r u_{0}}{8 a_{0}} \frac{u_{0}}{u_{0}+3 A_{0} d_{0}}}}+\phi_{3}\left(\varepsilon_{1}, \varepsilon_{2}\right)
\end{aligned}
$$




$$
\begin{gathered}
=\frac{\frac{r x_{12}^{2}}{K_{0}^{2}} \frac{3 A+2 x_{12}}{\left(A+x_{12}\right)^{2}} \varepsilon_{1}-\varepsilon_{2}}{\sqrt{\frac{r u_{0}}{8 a_{0}} \frac{u_{0}}{u_{0}+3 A_{0} d_{0}}}}+\phi_{3}\left(\varepsilon_{1}, \varepsilon_{2}\right), \\
\widetilde{L}_{23}\left(\varepsilon_{1}, \varepsilon_{2}\right)=1, \widetilde{\widetilde{L}}_{24}\left(\varepsilon_{1}, \varepsilon_{2}\right)=\frac{\frac{d_{0} r_{0}\left(u_{0}+10 A_{0} d_{0}\right)}{\left(u_{0}+2 A_{0} d_{0}\right)\left(u_{0}+3 A_{0} d_{0}\right)}}{\sqrt{\frac{r u_{0}}{8 a_{0}} \frac{u_{0}}{u_{0}+3 A_{0} d_{0}}}}+\phi_{4}\left(\varepsilon_{1}, \varepsilon_{2}\right),
\end{gathered}
$$

and $\phi_{i}(i=1,2,3,4)$ are smooth functions of $\left(\varepsilon_{1}, \varepsilon_{2}\right) ; \widetilde{\widetilde{R}}_{20}$ is a $C^{\infty}$ function in variables $\left(\varepsilon_{1}, \varepsilon_{2}\right)$ of at least the third order with respect to $(\mu, \nu)$, and the coefficients depend smoothly on $\varepsilon_{1}$ and $\varepsilon_{2}$.

Let $\widetilde{x}(t)=\mu(t)-\frac{1}{2} \phi_{2}\left(\varepsilon_{1}, \varepsilon_{2}\right), \widetilde{y}(t)=v(t)$. Then System (18) is $C^{\infty}$ equivalent to

$$
\left\{\begin{array}{l}
\dot{\widetilde{x}}(t)=\widetilde{y}(t), \\
\dot{\widetilde{y}}(t)=\nu_{1}+\lambda \nu_{2} \widetilde{y}(t)+\widetilde{x}^{2}(t)+\lambda \widetilde{x}(t) \widetilde{y}+Q\left(\widetilde{x}(t), \widetilde{y}(t), \varepsilon_{1}, \varepsilon_{2}\right),
\end{array}\right.
$$

where

$$
\begin{aligned}
& \nu_{1}=\frac{4 a_{0} x_{12}}{u_{0}} \varepsilon_{2}+\widehat{\phi}_{1}\left(\varepsilon_{1}, \varepsilon_{2}\right), \lambda=\frac{\frac{d_{0} r_{0}\left(u_{0}+10 A_{0} d_{0}\right)}{\left(u_{0}+2 A_{0} d_{0}\right)\left(u_{0}+3 A_{0} d_{0}\right)}}{\sqrt{\frac{r u_{0}}{8 a_{0} \frac{u_{0}}{u_{0}+3 A_{0} d_{0}}}}+\widehat{\phi}_{4}\left(\varepsilon_{1}, \varepsilon_{2}\right),} \\
& \nu_{2}=\frac{\frac{r x_{12}^{2}}{K_{0}^{2}} \frac{3 A+2 x_{12}}{\left(A+x_{12}\right)^{2}} \varepsilon_{1}-\varepsilon_{2}}{\lambda \sqrt{\frac{r u_{0}}{8 a_{0}} \frac{u_{0}}{u_{0}+3 A_{0} d_{0}}}}+\widehat{\phi}_{3}\left(\varepsilon_{1}, \varepsilon_{2}\right),
\end{aligned}
$$

and $\widehat{\phi}_{i}(i=1,2,3,4)$ are smooth functions of $\left(\varepsilon_{1}, \varepsilon_{2}\right) ; Q$ is a $C^{\infty}$ function in variables $\left(\varepsilon_{1}, \varepsilon_{2}\right)$ of at least the third order with respect to $(\widetilde{x}, \widetilde{y})$, and the coefficients depend smoothly on $\varepsilon_{1}$ and $\varepsilon_{2}$.

Since the above parameter transformation from $\left(\varepsilon_{1}, \varepsilon_{2}\right)$ to $\left(\nu_{1}, \nu_{2}\right)$ is not singular in a small neighborhood of $\left(\varepsilon_{1}, \varepsilon_{2}\right)=(0,0)$, by the Bogdanov-Takens theory (see [4], [5], [17]), System (14) is strongly topologically equivalent to

$$
\left\{\begin{array}{l}
\dot{\bar{x}}(t)=\bar{y} \\
\dot{\bar{y}}(t)=\nu_{1}+\lambda \nu_{2} \bar{y}+\bar{x}^{2}+\lambda \overline{x y} .
\end{array}\right.
$$

This proves Theorem 3.3 .

\section{Discussion}

In this paper, we introduce the weak Allee effect to the prey of System (2) studied by Ruan and Xiao [24]. We made a qualitative analysis of the model depending on all parameters and showed that the system exhibits some bifurcations.

First, we showed that the predator population $y$ will go extinct if the Allee effects constant $B$ satisfies $B \geq \frac{A_{1}^{2}}{A_{2}}$. In addition, the predator population also goes to extinction due to lack of the prey population when the prey carrying capacity $K$ is less than $\frac{d}{m a}$. 
Second, we showed that when $0<B<\frac{A_{1}^{2}}{A_{2}}$ and $K>\frac{d}{m a}$, the outcome of the survival depends on initial population sizes as is illustrated in Theorem 2.4. It was shown that the system undergoes a series of bifurcations including the saddle-node bifurcation, the supercritical and the subcritical Hopf bifurcations, and Bogdanov-Takens bifurcation. By combining mathematical analysis and numerical simulation, we have shown that the Allee effects may be a destabilizing force in predator-prey systems. Due to the weak Allee effects of the predator, the equilibrium of the system may change from asymptotically stable or neutral stable to unstable, to neutral stable, or the system will take much longer time to reach the stable state.

Although we have investigated qualitative analysis and bifurcations of the system, the global qualitative analysis is not determined explicitly. To investigate this problem, more complicated mathematical methods such as coincidence degree theory may be useful. We leave this for future work.

Acknowledgment. We would like extend our thanks to the anonymous referees for very helpful suggestions and comments which led to improvements of our original manuscript. We also thank professors Huaiping Zhu, Wendi Wang, Sze-Bi Hsu and Ying-Hen Hsieh for their helpful comments and constructive suggestions.

\section{References}

[1] W. C. Allee, Animal Aggregations, a Study in General Sociology, The University of Chicago Press, Chicago, IL., 1931.

[2] , The Social Life of Animals, Norton, New York, 1938.

[3] The Social Life of Animals, Revised Edition, Beacon Press, Boston, MA, 1958.

[4] R. Bogdanov, Bifurcations of a limit cycle for a family of vector fields on the plane, Selecta Math. Soviet. 1 (1981), 373-388.

[5] - Versal deformations of a singular point on the plane in the case of zero eigenvalues, Selecta Math. Soviet. 1 (1981), 389-421.

[6] C. W. Clark, Mathematical Bioeconomics, The Optimal Management of Renewable Resources, 2nd edn. John Wiley \& Sons Inc., New York, 1990.

[7] A. Deredec and F. Courchamp, Extinction thresholds in host-parasite dynamics, Ann. Zool. Fenn. 40 (2003), 115-130.

[8] C. W. Fowler and J. D. Baker, A review of animal population dynamics at extremely reduced population levels, Rep. Int. Whaling Comm. 41 (1991), 545-554.

[9] A. Kent, C. P. Doncaster, and T. Sluckin, Consequences for predators of rescue and Allee effects on prey, Ecol. Model. 162 (2003), 233-245.

[10] Y. Kuang and E. Beretta, Global qualitative analysis of a ratio-dependent predator-prey system, J. Math. Biol. 36 (1998), no. 4, 389-406.

[11] A. J. Lotka, Elements of Physical Biology, Williams \& Wilkins, Baltimore, 1926.

[12] L. Perko, Differential Equations and Dynamical Systems, Springer, New York, 1996.

[13] G. D. Ruxton, W. S. C. Gurney, and A. M. de Roos, Interference and generation cycles, Theoret. Population Biol. 42 (1992), 235-253.

[14] S. Schreiber, Allee effects, extinctions, and chaotic transients in simple population models, Theo. Pop. Biol. 64 (2003), no. 2, 201-209.

[15] P. A. Stephens and W. J. Sutherland, Consequences of the Allee effects for behaviour, ecology and conservation, Trends Ecol. Evol. 14 (1999), no. 10, 401-405. 
[16] P. A. Stephens, W. J. Sutherland, and R. P. Freckleton, What is the Allee effects?, Oikos 87 (1999), 185-190.

[17] F. Takens, Forced oscillations and bifurcations, Applications of global analysis, I (Sympos., Utrecht State Univ., Utrecht, 1973), pp. 1-59. Comm. Math. Inst. Rijksuniv. Utrecht, No. 3 - 1974, Math. Inst. Rijksuniv. Utrecht, Utrecht, 1974.

[18] V. Volterra, Fluctuations in the abundance of a species considered mathematically, Nature 118 (1926), 558-560.

[19] M. H. Wang and M. Kot, Speeds of invasion in a model with strong or weak Allee effects, Math. Biosci. 171 (2001), no. 1, 83-97.

[20] M. H. Wang, M. Kot, and M. G. Neubert, Integrodifference equations, Allee effects, and invasions, J. Math. Biol. 44 (2002), no. 2, 150-168.

[21] G. Wang, X. G. Liang, and F. Z. Wang, The competitive dynamics of populations subject to an Allee effect, Ecol. Model 124 (1999), no. 2-3, 183-192.

[22] D. Xiao, Bifurcations of saddle singularity of codimension three of a planar vector field with nilpotent linear part, Sci. Sinica A 23 (1993), 252-263.

[23] D. Xiao, L. Jennings, Bifurcations of a ratio-dependent predator-prey system with constant rate harvesting, SIAM J. Appl. Math. 65 (2005), no. 3, 737-753.

[24] S. Ruan and D. Xiao, Global analysis in a predator-prey system with nonmonotonic functional response, SIAM J. Appl. Math. 61 (2001), no. 4, 1445-1472.

[25] A. Yakubu, Multiple attractors in juvenile-adult single species models, J. Difference Equ. Appl. 9 (2003), no. 12, 1083-1098.

[26] Z. Zhang, T. Ding, W. Huang, and Z. Dong, Qualitative Theory of Differential Equations, Science Press, Beijing, 1992 (in Chinese). English edition: Transl. Math. Monogr., vol. 101, Amer. Math. Soc., Providence, RI, 1992.

[27] S. Zhou, Y. Liu, and G. Wang, The stability of predator-prey systems subject to the Allee effects, Theo. Pop. Biol. 67 (2005), 23-31.

[28] H. Zhu and S. A. Campbell, and G. S. K. Wolkowicz, Bifurcation analysis of a predatorprey system with nonmonotonic fuctional response, SIAM J. Appl. Math. 63 (2005), $636-682$.

RONGZHEN LIN

The Academy of Fundamental and Interdisciplinary Science

Harbin Institute of TEChNOLOGY

3041\#, 2 Yi-Kuang Street

Harbin, 150080, P. R. China

E-mail address: linrongzhen12345@yahoo.com.cn

SHEngQIANG LiU

The Academy of Fundamental and Interdisciplinary Science

HARBin InSTITUTE OF TEChNOLOGY

3041\#, 2 Yi-KuANG StREet

Harbin, 150080, P. R. China

E-mail address: sqliu@hit.edu.cn

XiAOHONG LAI

School of Mathematical Sciences

XIAMEN UNIVERSITY

Xiamen, 361005, P. R. China

E-mail address: wright19830305@yahoo.com.cn 